INPLASY

PROTOCOL

To cite: Xue et al. Efficacy and safety of vagus nerve stimulation for ischemic stroke: evidence from randomized controlled trials. Inplasy protocol 202180078. doi: 10.37766/inplasy2021.8.0078

Received: 20 August 2021

Published: 20 August 2021

Corresponding author: Tao Xue

2992326676@qq.com

Author Affiliation:

Department of Neurosurgery, Beijing Tiantan Hospital, Capital Medical University, Beijing, China.

Support: NSFC.

Review Stage at time of this submission: The review has not yet started.

Conflicts of interest:

None declared.

\section{Efficacy and safety of vagus nerve stimulation for ischemic stroke: evidence from randomized controlled trials}

Xue, $\mathrm{T}^{1}$; Chen, $\mathrm{S}^{2}$; Zhang, $\mathrm{J}^{3}$.

Review question / Objective: The aim of this meta-analysis of randomized controlled trials is to evaluate the efficacy and safety of VNS for ischemic stroke.

Condition being studied: Evidence from animal experiments suggests that vagus nerve stimulation combined with rehabilitation enhances forelimb function after experimental stroke and can improve task-specific plasticity. At present five clinical randomized controlled trials of vagus nerve stimulation (4 invasive and 1 non-invasive) paired with upper extremity rehabilitation have been done in patients who have suffered stroke. The results indicate that vagus nerve stimulation combined with rehabilitation is a novel strategy to help people achieve improvement in upper limb motor function after stroke.

INPLASY registration number: This protocol was registered with the International Platform of Registered Systematic Review and Meta-Analysis Protocols (INPLASY) on 20 August 2021 and was last updated on 20 August 2021 (registration number INPLASY202180078).

\section{INTRODUCTION}

Review question / Objective: The aim of this meta-analysis of randomized controlled trials is to evaluate the efficacy and safety of VNS for ischemic stroke.

Condition being studied: Evidence from animal experiments suggests that vagus nerve stimulation combined with rehabilitation enhances forelimb function after experimental stroke and can improve task-specific plasticity. At present five clinical randomized controlled trials of vagus nerve stimulation (4 invasive and 1 non-invasive) paired with upper extremity rehabilitation have been done in patients who have suffered stroke. The results 
indicate that vagus nerve stimulation combined with rehabilitation is a novel strategy to help people achieve improvement in upper limb motor function after stroke.

\section{METHODS}

Participant or population: Adults with ischemic stroke (as diagnosed by a clinician, or using any recognized diagnostic criteria) will be included.

Intervention: VNS was the main intervention.

Comparator: VNS + rehabilitation VS. rehabilitation alone.

\section{Study designs to be included: RCT.}

Eligibility criteria: The inclusion criteria were set up as follows: (1) type of study: RCT; (2) language restrictions: only available in English; (3) participating patients: adults patients with ischemic stroke; (4) intervention: VNS; and (5) outcomes: Fugl-Meyer Assessment-Upper Extremity (FMA-UE), Wolf Motor Function Test (WMFT; function and time score), Motor Activity Log (MAL), Stroke Impact Scale (SIS) score, Stroke Specific Quality of Life (SS-QOL), EQ-5D, the Beck Depression Inventory (BDI) and adverse events. Included RCTs were not requested to supply all the outcomes mentioned above. The exclusion criteria were set-up as follows: (a) types of study: retrospective studies, cohort studies, case reviews and case reports; (b) control: active control (i.e. that a known, effective treatment as opposed to a placebo is compared with an experimental treatment).

Information sources: Four main databases, MEDLINE, EMBASE, CENTRAL and ClinicalTrials.gov, were systematically searched by two authors (TX and SJC) using the following search strategy: (((VNS[Title/Abstract])) OR (vagus nerve stimulation[Title/Abstract])) AND (ischemic stroke[Title/Abstract]) for MEDLINE; 'VNS'/ $\exp$ OR 'vagus nerve stimulation'/exp AND 'ischemic stroke'/exp for EMBASE; "VNS" in Title Abstract Keyword OR "vagus nerve stimulation" in Title Abstract Keyword AND "ischemic stroke" in Title Abstract Keyword for CENTRAL; "VNS or vagus nerve stimulation | ischemic stroke" for ClinicalTrials.gov. Studies that matched the abstracts and titles were queried. In addition, two investigators (TX and SJC) ensured that all relevant studies and reports were included in this study. They independently and manually screened the list of references from the RCTs in August 2021.

Main outcome(s): Fugl-Meyer AssessmentUpper Extremity (FMA-UE) score.

Additional outcome(s): Wolf Motor Function Test (WMFT; function and time score), Motor Activity Log (MAL), Stroke Impact Scale (SIS) score, Stroke Specific Quality of Life (SS-QOL), EQ-5D, the Beck Depression Inventory (BDI) and adverse events.

Quality assessment / Risk of bias analysis: The risk-of-bias plot was assessed using Review Manager 5.3 software (The Cochrane Collaboration Collaboration, Oxford, UK) for individual studies. The unifed standard of the Cochrane Collaboration was applied to assess the risk of bias for RCTs, which included selection bias, performance bias, detection bias, attrition bias, reporting bias, and other potential biases.

Strategy of data synthesis: Two reviewers (TX and SJC) independently assessed study records in May 2020 from the reference lists of RCTs and electronic databases, according to the eligibility criteria previously mentioned. We excluded duplicates and research articles for which only the abstracts were available. When disagreements emerged among two reviewers, the disputed data were discussed with a third person (JGZ) who did not participate in the data collection process, to determine whether these data should be included in the study. After rigorous selection and evaluation of the literature by the two reviewers, all data were extracted from the RCTs as follows: basic information regarding the RCTs, 
outcome, inclusion and exclusion criteria, study design, and outcome assessments. The data were assessed using Review Manager 5.3 software (The Cochrane Collaboration), and the risk ratio (risk ratio [RR], 95\% confidence interval [CI]) was analyzed using dichotomous outcomes and calculated using a random-effect model. Heterogeneity was estimated using the 12 statistic: $12 \quad 50 \%$ denotes 'substantial heterogeneity'. We implemented subgroup analyses to detect different types of VNS. Sensitivity analysis was used to explore the stability of the consolidated results. A p value $<0.05$ was considered to be significant and two-tailed tests were performed for all the analyses.

Subgroup analysis: We implemented subgroup analyses to detect different types of VNS.

Sensitivity analysis: Sensitivity analysis was used to explore the stability of the consolidated results.

Language: English.

Country(ies) involved: China.

Keywords: VNS; ischemic stroke; rehabilitation; upper limb motor function.

Contributions of each author:

Author 1 - Tao Xue.

Author 2 - Shujun Chen.

Author 3 - Jianguo Zhang. 